\title{
Studies of Li intercalation of hydrogenated graphene on $\mathrm{SiC}(0001)$
}

Somsakul Watcharinyanon, Leif Johansson, A. A. Zakharov and Chariya Virojanadara

\section{Linköping University Post Print}

N.B.: When citing this work, cite the original article.

Original Publication:

Somsakul Watcharinyanon, Leif Johansson, A. A. Zakharov and Chariya Virojanadara, Studies of Li intercalation of hydrogenated graphene on SiC(0001), 2012, Surface Science, (606), 3-4, 401-406.

http://dx.doi.org/10.1016/j.susc.2011.10.023

Copyright: Elsevier

http://www.elsevier.com/

Postprint available at: Linköping University Electronic Press

http://urn.kb.se/resolve?urn=urn:nbn:se:liu:diva-73546 


\title{
Studies of Li intercalation of hydrogenated graphene on $\mathrm{SiC}(0001)$
}

\author{
S. Watcharinyanon ${ }^{1}$, L. I. Johansson ${ }^{1}$, A. A. Zakharov ${ }^{2}$, and C. Virojanadara ${ }^{1}$ \\ ${ }^{1}$ Department of Physics, Chemistry, and Biology, Linköping University, S-58183 \\ Linköping, Sweden \\ ${ }^{2}$ MAX-lab, Lund University, S-22100, Lund, Sweden
}

\begin{abstract}
The effects of $\mathrm{Li}$ deposition on hydrogenated bilayer graphene on $\mathrm{SiC}(0001)$ samples, i.e. on quasi-freestanding bilayer graphene samples is studied using low energy electron microscopy, micro-low-energy electron diffraction and photoelectron spectroscopy. After deposition, some $\mathrm{Li}$ atoms form islands on the surface creating defects that are observed to disappear after annealing. Some other $\mathrm{Li}$ atoms are found to penetrate through the bilayer graphene sample and into the interface where $\mathrm{H}$ already resides. This is revealed by the existence of shifted components, related to $\mathrm{H}-\mathrm{SiC}$ and $\mathrm{Li}-\mathrm{SiC}$ bonding, in recorded core level spectra. The Dirac point is found to exhibit a rigid shift to about $1.25 \mathrm{eV}$ below the Fermi level, indicating strong electron doping of the graphene by the deposited $\mathrm{Li}$. After annealing the sample at $300-400{ }^{\circ} \mathrm{C}$ formation of $\mathrm{LiH}$ at the interface is suggested from the observed change of the dipole layer at the interface. Annealing at $600{ }^{\circ} \mathrm{C}$ or higher removes both $\mathrm{Li}$ and $\mathrm{H}$ from the sample and a monolayer graphene sample is reestablished. The $\mathrm{Li}$ thus promotes the removal of $\mathrm{H}$ from the interface at a considerably lower temperature than after pure $\mathrm{H}$ intercalation.
\end{abstract}

\section{Introduction}

Graphene has attracted intense recent interest due to its unique two-dimensional electron gas and electron transport properties [1-3]. This makes graphene a promising material for future carbon-based electronic devices. Epitaxial growth of graphene on silicon carbide (SiC) was proposed as the way to fabricate high quality graphene with a large homogeneous area [4-6]. In addition, the epitaxial growth on the Si-terminated $\mathrm{SiC}(0001)$ substrate is known to allow preparation of large homogeneous graphene samples with a thickness down to a single monolayer [4-5, 7]. On the C-terminated $\mathrm{SiC}(000 \overline{1})$ substrate [8] it has been difficult to control the number of graphene layers. However, for epitaxial 
graphene prepared on $\operatorname{SiC}(0001)$, a carbon $(6 \sqrt{3} \times 6 \sqrt{3}) \mathrm{R} 30^{\circ}$ reconstructed layer having no graphitic electronic properties, and acting as a buffer layer, is present at the interface between graphene and SiC. This layer exhibits a large band gap and a Fermi level pinned by a state related to the dangling bonds of $\mathrm{Si}$ atoms in the $\mathrm{SiC}$ bilayer [9]. Therefore this buffer layer is considered to be a main obstacle to the development of future electronic devices based on graphene grown on $\mathrm{SiC}(0001)$. Nonetheless, earlier studies have demonstrated that this buffer layer can be decoupled from the substrate and transformed into a graphene layer by hydrogen $(\mathrm{H})$ [6, 10-12], gold $(\mathrm{Au})$ [13], oxygen $\left(\mathrm{O}_{2}\right)$ [14] and lithium (Li) [15-16] intercalation.

For the hydrogen case, it has been demonstrated [6, 10-12] that hydrogen intercalates the buffer layer and stays chemically inert at the interface even if the sample is stored for months in an atmospheric ambient. A temperature of well above $700{ }^{\circ} \mathrm{C}[6,10-12]$ is required to remove the hydrogen, which is quite a high temperature for hydrogen storage applications.

In the present work, we study the effects of $\mathrm{Li}$ deposition on hydrogenated monolayer graphene samples, using low energy electron microscopy (LEEM), micro-low-energy electron diffraction ( $\mu$-LEED), photoelectron spectroscopy (PES) and angle resolved photoelectron spectroscopy (ARPES). Surprisingly, we find that Li can remove the hydrogen from the interface region already at a relatively low temperature $\left(300{ }^{\circ} \mathrm{C}\right)$ and partially form a $\mathrm{LiH}$ compound between the carbon layers and the $\mathrm{SiC}$ substrate. This is very interesting since the $\mathrm{LiH}$ has been claimed to have the highest hydrogen content possibly of any hydride [17-18]. Moreover, Li contributes to an electron doping of the graphene, which is also of interest for some specific applications.

\section{Experimental}

The epitaxial graphene was prepared on $n$-type on-axis $6 \mathrm{H}-\mathrm{SiC}(0001)$ substrates with a mis-orientation error within $0.06^{\circ}$. Substrates were cut from wafers, purchased from SiCrystal, with chemical and mechanical polishing on the $\mathrm{Si}$ face. Before graphene formation the substrates were cleaned using the standard Radio Corporation of America 
(RCA) cleaning procedure [19] to remove organic and inorganic contaminations and etched in HF to remove surface oxide. The growth of graphene was performed by direct current heating the substrate at a temperature of $1300^{\circ} \mathrm{C}$ for a few minutes in in situ at a base pressure of approximately $10^{-10}$ mbar. This is known to produce graphene samples with a dominant coverage of $1 \mathrm{ML}$. Then, the samples were exposed to $\mathrm{H}$ using an atomic hydrogen source. The samples were annealed at a temperature of $700{ }^{\circ} \mathrm{C}$ and exposed to atomic hydrogen at a pressure of about $1 \times 10^{-6}$ mbar. In this process, $\mathrm{H}$ intercalates through the graphene and the buffer layer and bond to $\mathrm{Si}$ atoms at the substrate interface [10-12]. Upon $\mathrm{H}$ intercalation, the buffer layer is lifted up and transformed into a second graphene layer. The monolayer graphene is therefore converted into hydrogenated bilayer graphene. It deserves to be noted that the amount of intercalated $\mathrm{H}$ on the samples is different due to differences in the exposure time and instrument utilized. The sample studied in LEEM is fully hydrogenated while the sample studied by PES is partly hydrogenated.

On these hydrogenated bilayer graphene samples Li was deposited using a SAES getter source. The samples were kept at room temperature and exposed to $\mathrm{Li}$ atoms for a few minutes. The morphology and electronic structure of the hydrogenated bilayer graphene samples was studied before and after Li depositions and also after subsequent annealing at temperatures from 300 to $900{ }^{\circ} \mathrm{C}$. Typically, the temperature measured across the sample varies less than $20^{\circ} \mathrm{C}$. LEEM, $\mu$-LEED, ARPES and PES were utilized at beamline I311 [20] and I4 [21] at the MAX-lab synchrotron radiation facility in Lund, Sweden. Beamline I311 is equipped with a modified SX700 monochromator, which provides light for two end stations. The first station is equipped with large Scienta electron analyzer. It was utilized for high-resolution studies of the C 1s, Si 2p, Li 1s core levels and the valence bands. The core-level spectra were acquired at normal emission with a light incidence angle of $40^{\circ}$. The total energy resolution was set to $\leq 0.10 \mathrm{eV}$ for photon energies from 33 to $450 \mathrm{eV}$ and to $\leq 0.30 \mathrm{eV}$ for energies up to $750 \mathrm{eV}$. The second station is equipped with a spectroscopic photoemission LEEM (SPELEEM) instrument. The spatial resolution of this microscope is better than $10 \mathrm{~nm}$ in the LEEM mode. Beamline I4 is equipped with a spherical grating monochromator and an angular 
resolved electron energy analyzer with a two-dimensional detector from SPECS. The low angular dispersion lens mode which provides an acceptance angle of $\pm 7^{\circ}$ was selected for detailed ARPES studies of the valence band around the K-point in the Brillouin zone. An incidence angle between the incoming light and the detector of $45^{\circ}$ was chosen as the measurement geometry. The base pressure was about $10^{-10}$ mbar in both the experimental end stations utilized.

\section{Results and Discussion}

Figs. 1a)-c) illustrate the surface morphology of the in situ as grown monolayer graphene, after hydrogenation, and after Li deposition. Figs. 1d)-f) show the corresponding LEED patterns. Before and after hydrogenation, Fig. 1a) and b), the surface morphology appears very homogeneous and no significant changes are observed in the mirror and LEEM images. In the $\mu$-LEED pattern, on the other hand, clear differences are visible i.e. only the $(1 \times 1)$ graphene spots without any contribution from the buffer layer, $(6 \sqrt{ } 3 \times 6 \sqrt{ } 3) R 30^{\circ}$, are observed after $\mathrm{H}$ exposure, cf. Fig. 1d) and 1e). This result indicates that the buffer layer is eliminated after $\mathrm{H}$ exposure, in agreement with earlier observations.[6, 10-12] $\mathrm{Li}$ was then deposited on the sample at room temperature. A rougher surface and small dark islands appear directly after Li deposition as illustrated by the mirror image in Fig. 1c). The former is suggested to be due to distortions in the graphene layer induced by tension caused by Li atoms [16]. The latter show Li droplets formed on the surface as suggested earlier after $\mathrm{Li}$ deposition on as grown monolayer graphene [15], i.e. on nonhydrogenated samples. These droplets reduced in size upon annealing and were no longer observable after annealing at about $220^{\circ} \mathrm{C}$. In addition to this a $(\sqrt{ } 3 \times \sqrt{3}) R 30^{\circ}$ reconstructed surface developed directly after Li deposition, see Fig. 1f). This pattern has earlier been suggested to originate from Li that partly occupy the graphene $\pi$ bonds on the surface and form a $\mathrm{C}_{6} \mathrm{Li}$ compound [22]. It has been demonstrated that the formation of $\mathrm{C}_{6} \mathrm{Li}$ gives rise to a folding of the energy bands so the $\pi$-bands then appear close to the Fermi level at the $\bar{\Gamma}$-point in the ARPES spectra collected at a photon energy of $41 \mathrm{eV}$ [23]. However, we do not observe any folded $\pi$-band at the $\Gamma$-point in our ARPES spectra collected at the different photon energy $(33 \mathrm{eV})$. Therefore the photoionization cross section different may prevent us from observing this contribution or the 
$(\sqrt{ } 3 \times \sqrt{3}) R 30^{\circ}$ superstructure observed in the LEED pattern taken directly after Li deposition may instead arise from ordered $\mathrm{Li}$ on the sample.

For thickness identification, recorded electron reflectivity curves $(I-V)$ are used and plotted in Fig 2. The $I-V$ curves collected from the monolayer graphene sample before and after $\mathrm{H}$ exposure are displayed in Fig. 2a) and 2b). These are in agreement with earlier results [11] and show transformation from monolayer graphene (one dip) to bilayer graphene (two dips). After Li deposition (Fig. 2c), two minima are still visible. The work function of the sample has decreased as seen from the shift of the reflectivity onset to lower electron energy. The separation between the minima increases. This increase in separation was earlier suggested [15] to be caused by the changes in the electron diffraction path induced by the $\mathrm{Li}$ atoms that go in between the graphene and the buffer layer and into the interface. Apart from this the first minimum is somewhat broader compared to the earlier observation after $\mathrm{Li}$ intercalation [15]. This may be an effect from an inhomogeneous intercalation or disorder in the interface region either by $\mathrm{H}-$ or Li- atoms. It should be noted that there is no significant difference observed between the $I-V$ curves detected from the bright or dark areas after Li deposition. A significant change is, however, observed after heating the sample to $600{ }^{\circ} \mathrm{C}$ when the collected $I-V$ curve again shows only one dip that represents monolayer graphene, see Fig. 2d). This is very interesting since the intercalated $\mathrm{H}$ was reported to be stable at the graphene-SiC interface up to a temperature of about $850{ }^{\circ} \mathrm{C}$ [6, 10-12]. Therefore, the $\mathrm{Li}$ atoms seem to play an important role for the change of the hydrogenated bilayer graphene. Furthermore, the $I-V$ curve after annealing at $600{ }^{\circ} \mathrm{C}$ is still shifted to the lower electron energy compared to the as grown monolayer graphene. These observations suggest that $\mathrm{Li}$ is still present on the sample, which lower the work function of the sample.

Core-level PES was utilized to further investigate the influence of $\mathrm{Li}$ deposition on hydrogenated bilayer graphene by probing the $\mathrm{C} 1 \mathrm{~s}, \mathrm{Si} 2 \mathrm{p}$, and $\mathrm{Li} 1 \mathrm{~s}$ core-level electrons. Fig. 3a) illustrates a series of $\mathrm{C} 1 \mathrm{~s}$ core-level spectra collected at a photon energy of 450 $\mathrm{eV}$. The $\mathrm{C} 1 \mathrm{~s}$ spectrum of the as grown monolayer graphene (bottom curve) consists of three components, i.e., bulk $\mathrm{SiC}$, graphene (G) and buffer layer (B). After partial 
hydrogenation, the buffer layer component is significantly reduced, as displayed in the middle curve. However, the $(6 \sqrt{3} \times 6 \sqrt{3}) R 30^{\circ}$ buffer layer spots are still detectable in the LEED pattern, but are very faint. The $\mathrm{G} / \mathrm{SiC}$ intensity ratio in the $\mathrm{C} 1$ s spectrum is found to increase after hydrogenation suggesting an increase of graphene in the sample. At the same time the bulk $\mathrm{SiC}$ component (now labeled $\mathrm{SiC}^{\prime}$ ) is shifted about $0.2 \mathrm{eV}$ to lower binding energy after $\mathrm{H}$ exposure. This has been suggested to be due to the formation of a dipole layer at the interface caused by $\mathrm{H}-\mathrm{SiC}$ substrate interaction [12]. However, a shift of about $0.7 \mathrm{eV}$ of the $\mathrm{SiC}$ bulk component has been observed earlier on a fully hydrogenated graphene [12]. The smaller shift observed in this case is the result of incomplete hydrogenation.

The spectrum of the hydrogenated bilayer graphene after Li deposition is illustrated in the top panel of Fig. 3a). An extra component (labeled $\mathrm{SiC}^{\prime \prime}$ ) is observed at the low binding energy side. This component is shifted by about $2 \mathrm{eV}$ compared to the $\mathrm{SiC}$ component on the as grown sample. This shifted $\mathrm{SiC}^{\prime \prime}$ component is therefore interpreted to arise from $\mathrm{Li}$ atoms that have penetrated the graphene layers and interact with the $\mathrm{SiC}$ substrate forming dipoles at the interface region. An additional small shoulder is detected on the higher binding energy tail of the spectrum. This shoulder is most probably related to the changes in the coulomb charge density in the interface region [15]. The G peak shows a slight shift to higher binding energy. The shift is suggested to be due to the charge transfer from $\mathrm{Li}$ to graphene. Moreover, an increase of the $\mathrm{B} /$ "total $\mathrm{SiC}$ " intensity ratio is observed after $\mathrm{Li}$ deposition. This is interesting since one may expect the $\mathrm{Li}$ atoms to intercalate more at the interface and transform the remaining buffer layer into second graphene layer. However this $\mathrm{C} 1 \mathrm{~s}$ spectrum shows the opposite. The increase of the ratio implies a recreation of buffer layer domains after $\mathrm{Li}$ deposition. The $\mathrm{Li}$ atoms seem to penetrate into the hydrogenated areas, i.e. buffer layer free bilayer graphene domains, and interact with the $\mathrm{H}$ at the interface. This interaction removes the $\mathrm{H}$ from the interface and gives rise to a transformation of graphene into buffer layer again. Beside this, the ratio between $\mathrm{G}$ and total $\mathrm{SiC}$ signal is seen to decrease after $\mathrm{Li}$ deposition. We can therefore suggest that the graphene layer is partly destroyed after Li deposition, probably due to tension induced by $\mathrm{Li}$ atoms in the surface region [15]. However, after $\mathrm{Li}$ 
deposition, the $(6 \sqrt{3} \times 6 \sqrt{3}) R 30^{\circ}$ buffer layer spots are no longer detectable in the diffraction pattern, as illustrated by Fig 1f. The disappearance of the $(6 \sqrt{3} \times 6 \sqrt{ } 3) R 30^{\circ}$ spots may be caused by a disorder of the buffer layer induced after the Li deposition.

The sample was then annealed at different temperatures and the C1s spectra recorded are shown in Fig. 3b). Already at $300{ }^{\circ} \mathrm{C}$ the intensity of the B component is dramatically reduced. This shows that annealing improves the $\mathrm{Li}$ intercalation so more of the buffer layer is transformed into a graphene layer. For $\mathrm{Li}$ intercalation of monolayer graphene it has earlier been demonstrated that a more even $\mathrm{Li}$ distribution and intercalation is obtained after annealing to a few hundred degree celsius [15-16]. The bulk SiC component is now located at a binding energy in between the $\mathrm{SiC}^{\prime}$ and $\mathrm{SiC}^{\prime \prime}$ components indicating a change of the dipole layer at the interface. Some of the $\mathrm{Li}$ atoms are suggested to intercalate into the non-hydrogenated areas and transform the buffer layer to a graphene layer. Some other intercalate into the hydrogenated parts, and either react with or expel $\mathrm{H}$ atoms at or from the interface. The $\mathrm{H}$ atoms most probably bond to $\mathrm{Li}$ atom and form $\mathrm{LiH}$ since the $\mathrm{C}$ 1s spectra indicate that the interface dipole layer is changed. Only one bulk $\mathrm{SiC}$ component is now observed and with an energy separation to the $\mathrm{G}$ component that is $0.7 \mathrm{eV}$ smaller than after intercalation with pure $\mathrm{Li}$. The $\mathrm{Li} 1 \mathrm{~s}$ core level spectra shown and discussed below also support formation of $\mathrm{LiH}$. After annealing at $500{ }^{\circ} \mathrm{C}$, the bulk $\mathrm{SiC}$ component is seen to have shifted back to a higher binding energy. This indicates that the Li starts to leave the interface at this temperature. Annealing at 600 ${ }^{\circ} \mathrm{C}$ results in a further shift of the bulk $\mathrm{SiC}$ component and an increase in relative intensity of the B component. It is however not until after annealing at $900{ }^{\circ} \mathrm{C}$ that the shape of the $\mathrm{C} 1 \mathrm{~s}$ spectrum from the as grown monolayer graphene is completely restored. This result is in agreement with the LEEM observation, that only one dip is observed after annealing at $600{ }^{\circ} \mathrm{C}$, see Fig. 2d). This shows that $\mathrm{Li}$ and $\mathrm{H}$ are removed from the substrate interface and the bilayer graphene is transformed back into monolayer graphene.

A set of Si $2 p$ spectra acquired using a photon energy of $190 \mathrm{eV}$ is presented in Fig. $4 \mathrm{a}$. The spectrum of the as grown monolayer graphene (bottom curve) contain mainly contribution from the bulk $\mathrm{SiC}$ substrate. After $\mathrm{H}$ exposure, the monolayer graphene 
transforms partly into hydrogenated quasi-free standing bi-layer graphene. As seen in the middle curve, the bulk $\mathrm{SiC}$ component (now labeled $\mathrm{SiC}^{\prime}$ ) then shifts by about $0.2 \mathrm{eV}$ to lower binding energy. This corresponds well to the shift of the bulk SiC component observed in the $\mathrm{C}$ 1s spectrum after partial hydrogenation. The Si 2 p spectrum shows a shoulder at the lower binding energy and labeled $\mathrm{S}^{\prime}$. This shoulder originates from the interaction between $\mathrm{H}$ and $\mathrm{Si}$ atoms at the graphene-SiC interface resulting in $\mathrm{Si}-\mathrm{H}$ bonds. The effect of Li deposition on the hydrogenated bilayer graphene is clearly observed in $\mathrm{Si}$ $2 \mathrm{p}$ spectrum (top curve). Two additional doublets (labeled $\mathrm{SiC}^{\prime \prime}$ and $\mathrm{S}^{\prime \prime}$ ) are now detected at lower binding energy. Both doublets are found to shift by about $2 \mathrm{eV}$ compared to what was observed on the as grown sample. This is consistent with the shift observed of the bulk $\mathrm{SiC}$ component in the $\mathrm{C} 1$ s spectrum. The $\mathrm{SiC}^{\prime \prime}$ and $\mathrm{S}^{\prime \prime}$ components indicate the formation of a dipole layer at the graphene-SiC interface upon $\mathrm{Li}$ intercalation. The observed two set of doublets ( $\mathrm{SiC}^{\prime}, \mathrm{S}^{\prime}$ and $\left.\mathrm{SiC}^{\prime \prime}, \mathrm{S} 1^{\prime \prime}\right)$ confirm the existence of both $\mathrm{H}$ and $\mathrm{Li}$ at the uppermost $\mathrm{Si}-\mathrm{C}$ bilayer after $\mathrm{Li}$ deposition.

The evolution of the Si 2 p spectrum recorded after successive annealing from 300 to 900 ${ }^{\circ} \mathrm{C}$ is shown in Fig. 4b. Already after annealing at $300{ }^{\circ} \mathrm{C}$ the spectrum seems to contain essentially only two components with a dominant bulk $\mathrm{SiC}$ component located in between the $\mathrm{SiC}^{\prime}$ and $\mathrm{SiC}^{\prime \prime}$ components present before annealing. This confirms that the dipole layer at the interface is changed. We interpret that $\mathrm{Li}-\mathrm{Si}$ and $\mathrm{H}-\mathrm{Si}$ interactions dominate before annealing while $\mathrm{LiH}-\mathrm{Si}$ interactions dominate after annealing at 300 and $400{ }^{\circ} \mathrm{C}$. The Si $2 \mathrm{p}$ peaks have moved back to the initial position after annealing at $500{ }^{\circ} \mathrm{C}$ and higher temperature which shows that $\mathrm{Li}$ and $\mathrm{H}$ then have been essentially removed from the interface.

Li 1s spectra recorded from the hydrogenated bilayer graphene after Li deposition and annealing cycles are presented in Fig. 5. After deposition, the $\mathrm{Li} 1 \mathrm{~s}$ spectrum is decomposed into five components and denoted L1-L5. Our earlier study [15] of Li deposited on monolayer graphene suggested that the L1 component at a binding energy of $54.2 \mathrm{eV}$ is assigned to the ordered Li that partially occupies the graphene $\pi$ bonds and form a $(\sqrt{3} \times \sqrt{3}) \mathrm{R} 30^{\circ}$ superstructure on the surface. The L2 component at a binding 
energy of $54.7 \mathrm{eV}$ is related to $\mathrm{Li}$ atoms at the interface that bond to $\mathrm{Si}$ atoms in the topmost Si-C bilayer. The L3 and L4 components at binding energies of 56.0 and $56.8 \mathrm{eV}$ correspond to $\mathrm{Li}$ atoms interacting with the buffer layer and $\mathrm{Li}$ atoms in between the graphene sheet and the buffer layer, respectively. The additional component L5 which was not observed in the earlier study [15] is assigned to the Li that interacts with the $\mathrm{H}$ and forms $\mathrm{LiH}[24]$.

Changes are clearly distinguished after annealing. The intensities of the L1 and L4 components reduce dramatically already after annealing at $300{ }^{\circ} \mathrm{C}$. The attenuation of the L1 signal corresponds well to the decrease of the Li islands on the surface observed in the LEEM image after annealing to a few hundred degrees. At this temperature, the Li intercalation is known to improve [15-16]. The L2, L3 and L5 components still remain after annealing up to $400{ }^{\circ} \mathrm{C}$, confirming the existence of $\mathrm{Li}$ at the interface and formation of $\mathrm{LiH}$. After annealing at $500{ }^{\circ} \mathrm{C}$, the $\mathrm{Li} 1 \mathrm{~s}$ spectrum becomes broader since an additional component appears on the low binding energy side. This component is interpreted to correspond to $\mathrm{Li}$ on the surface since its relative intensity is highest at this low photon energy. We therefore suggest that $\mathrm{Li}$ has been pushed away from the interface and on to the graphene surface after annealing at $500{ }^{\circ} \mathrm{C}$. At $600{ }^{\circ} \mathrm{C}$, the $\mathrm{Li} 1 \mathrm{~s}$ signal is very weak showing that most of the $\mathrm{Li}$ is removed from the sample and at $900{ }^{\circ} \mathrm{C}$ no $\mathrm{Li}$ signal is detectable.

The influence of Li deposition on the electronic structure of the hydrogenated bilayer graphene was studied by ARPES. The band structure of graphene at the $\bar{K}$ point in the hexagonal Brillouin zone collected perpendicular to $\bar{\Gamma} \rightarrow \bar{K}$ direction is shown in Fig. 6 . A single $\pi$-band is observed before and two $\pi$-bands after hydrogenation, respectively, as displayed in Fig. 6a) and b). For the as grown monolayer graphene, the Dirac crossing point of the $\pi$ band is located around $0.4 \mathrm{eV}$ below the Fermi level indicating n-type doping due to the charge transfer from $\mathrm{SiC}$ substrate [25]. However, after hydrogenation the Dirac point is found to move towards the Fermi level. This indicates that the intercalated $\mathrm{H}$ decouples the graphene from the substrate and weakens the graphenesubstrate interaction. Directly after Li deposition, Fig. 6c) one broad $\pi$-band is observed, 
instead of two well-separated $\pi$-bands. This can be caused by distortion in the graphene layer induced by partial $\mathrm{Li}$ intercalation. The $\mathrm{Li}$ atoms penetrate into the interface, interact with the $\mathrm{H}$ atoms at the interface and give rise to a partial transformation of the graphene back to a carbon buffer layer. Contribution from Li atoms residing on top and below the uppermost graphene layer can explain the broad single $\pi$-band as arising from a strong doping of the graphene layers so they appear to consist of predominantly $1 \mathrm{ML}$ domains. The Dirac point exhibits a rigid shift further away from the Fermi level by about $1.25 \mathrm{eV}$ which corresponds to a charge concentration of about $1.1 \times 10^{14} \mathrm{~cm}^{-2}$. This indicates an additional electron doping of the graphene from the deposited $\mathrm{Li}$, i.e. that electrons from the alkali metal are released and injected into empty states in the $\pi$-band of the graphene layer. The most recent studies on $\mathrm{K}$ deposited graphene formed on hydrogenated $\mathrm{SiC}(0001)$ [26-27] has demonstrated that there is no gap opening at the $\bar{K}$ point which is consistent to our $\mathrm{Li}$ case. Instead they observed a plasmaronic dispersion, i.e. a diamond-like shape, near the Dirac energy. A shadow band next to the main band was also detected and suggested to be a plasmaron band [26-27]. We observe no pronounced deformation of the $\pi$-band around the Dirac point after Li deposition. This can be due to the high doping concentration obtained which reduces the strength of the plasmaron band [27]. After heating the sample to $300^{\circ} \mathrm{C}$ two $\pi$-bands are clearly visible as seen in Fig 6d). This is clear evidence of that more $\mathrm{Li}$ intercalation occurs upon heating, that more of the buffer layer transforms into a second graphene layer. The Dirac point is seen to move slightly up towards the Fermi level compared to before heating. Moreover, the bands near the Dirac point is clearly seen to be deformed, i.e. to show some curvature around the Dirac point. No shadow band near the main band is however resolvable and the diamond shape is less distinct than for K-doped graphene [26-27]. When increasing the temperature to $400{ }^{\circ} \mathrm{C}$, Fig. $6 \mathrm{e}$ ), two $\pi$-bands are still visible but the Dirac point now moves up closer to the Fermi level, i.e. the carrier concentration becomes lower due to desorption of $\mathrm{Li}$ atoms. After annealing at $600{ }^{\circ} \mathrm{C}$, a single $\pi$-band reappears but somewhat broader than on the initial surface since not all Li has desorbed at this temperature. The Dirac point is still slightly shifted to lower binding energy. This observation agrees well with the observed single dip in the $I-V$ curve after annealing at $600^{\circ} \mathrm{C}$ and that the dip is slightly shifted to lower electron energy compared to the as 
grown graphene, see Figs 2 d) and a). Further annealing at $800{ }^{\circ} \mathrm{C}$ shifts the Dirac point to the initial energy but the $\pi$-band is still broader than initially. This can be caused by that $\mathrm{Li}$ adsorption/intercalation and desorption creates defects and increased disorder in the graphene layer.

\section{Summary}

We have studied the effects of Li deposition on hydrogenated bilayer graphene samples, i.e. on quasi-free standing bilayer graphene samples. Hydrogenation of monolayer graphene on $\mathrm{SiC}(0001)$ [6, 11-12] transforms the buffer layer into a second graphene layer and shifts the Dirac point upwards to the Fermi level, forming quasi-free standing bilayer graphene. The effects induced by $\mathrm{Li}$ deposited on such samples at room temperature and by subsequent annealing at different temperatures are reported. After deposition part of the Li atoms are found to form droplets, occupy graphene $\pi$ bonds and form a $(\sqrt{3} \times \sqrt{3}) \mathrm{R} 30^{\circ}$ superstructure on the graphene surface. For the partly hydrogenated sample some $\mathrm{Li}$ is found to intercalate into the interface region, giving rise to a larger shift of the $\mathrm{SiC}$ substrate peaks and also a partial transformation of graphene back to buffer layer (peak B) carbon. At this stage the LEEM $I-V$ curves show two dips indicating bilayer graphene. However, the ARPES data shows only one broad $\pi$ band at the $\bar{K}$ point in the band structure, suggesting a sample with predominantly monolayer graphene coverage. The deposited $\mathrm{Li}$ atoms provide strong electron doping of the graphene layers, lowering the Dirac point to about $1.25 \mathrm{eV}$ from the Fermi level. After annealing at 300$400{ }^{\circ} \mathrm{C}$ the buffer layer (B) peak decreases and the energy separation between the bulk $\mathrm{SiC}$ and the graphene $(\mathrm{G})$ components in the $\mathrm{C} 1 \mathrm{~s}$ spectra is found to become smaller, compared to what was obtained after pure Li intercalation of monolayer graphene [15], i.e. on non-hydrogenated samples. This indicates that the interface dipole layer is different in these two cases. We suggest formation of $\mathrm{LiH}$ at the interface to be the reason for this. Also the $\mathrm{Li} 1 \mathrm{~s}$ and $\mathrm{Si} 2 \mathrm{p}$ core level results presented are consistent with formation of $\mathrm{LiH}$ at the interface and confirm that additional $\mathrm{Li}$ intercalation occurs upon annealing. Two $\pi$-bands are clearly visible at this stage and the Dirac point has started to shift back towards the Fermi level, indicating a decrease in the electron doping concentration of the graphene layers. When annealing the sample at $600{ }^{\circ} \mathrm{C}$ or higher 
temperature, the $\mathrm{Li}$ and $\mathrm{H}$ atoms are removed from the sample. This is very interesting since intercalated $\mathrm{H}$ was earlier reported to be stable at the graphene-SiC interface up to a temperature of about $850{ }^{\circ} \mathrm{C}[6,10-12]$. Therefore, the $\mathrm{Li}$ atoms appear to play an important role for the changes observed for the hydrogenated bilayer graphene samples.

\section{References}

[1] K.S. Novoselov, A.K. Geim, S.V. Morozov, D. Jiang, M.I. Katsnelson, I.V. Grigorieva, S.V. Dubonos, A.A. Firsov, Nature 438 (2005) 197.

[2] A.K. Geim, K.S. Novoselov, Nat. Mater. 6 (2007) 183.

[3] C. Berger, Z. Song, T. Li, X. Li, A.Y. Ogbazghi, R. Feng, Z. Dai, A.N. Marchenkov, E.H. Conrad, P.N. First, W.A. de Heer, J. Phys. Chem. B 108 (2004) 19912.

[4] C. Virojanadara, M. Syväjärvi, R. Yakimova, L.I. Johansson, A.A. Zakharov, T. Balasubramanian, Phys. Rev. B 78 (2008) 245403.

[5] C. Virojanadara, R. Yakimova, J.R. Osiecki, M. Syväjärvi, R.I.G. Uhrberg, L.I. Johansson, A.A. Zakharov, Surf. Sci. 603 (2009) L87.

[6] C. Virojanadara, R. Yakimova, A.A. Zakharov, L.I. Johansson, J. Phys. D 43 (2010) 374010 .

[7] R. Yakimova, C. Virojanadara, D. Gogova, M. Syväjärvi, D. Siche, K. Larsson, L.I. Johansson, Mater. Sci. Forum. 645-648 (2010) 565.

[8] U. Starke, C. Riedl, J. Phys.:condend. Matter 21 (2009) 134016.

[9] C. Berger, X. Wu, P.N. First, E.H. Conrad, X. Li, M. Sprinkle, J. Hass, F. Varchon, L. Magaud, M.L. Sadowski, M. Potemski, G. Martinez, W.A. de Heer, Adv. Solid State Phys. 47 (2008) 145.

[10] C. Riedl, C. Coletti, T. Iwasaki, A.A. Zakharov, U. Starke, Phys. Rev. Lett. 103 (2009) 246804.

[11] C. Virojanadara, A.A. Zakharov, R. Yakimova, L.I. Johansson, Surf. Sci. 604 (2010) L4.

[12] S. Watcharinyanon, C. Virojanadara, J.R. Osiecki, A.A. Zakharov, R. Yakimova, R.I.G. Uhrberg, L.I. Johansson, Surf. Sci. 605 (2011) 1662.

[13] I. Gierz, T. Suzuki, R.T. Weitz, D.S. Lee, B. Krauss, C. Riedl, U. Starke, H. Höchst, J.H. Smet, C.R. Ast, K. Kern, Phys. Rev. B 81 (2010) 235408. 
[14] S. Oida, F.R. McFeely, J.B. Hannon, R.M. Tromp, M. Copel, Z. Chen, Y. Sun, D.B. Farmer, J. Yurkas, Phys. Rev. B 82 (2010) 041411.

[15] C. Virojanadara, S. Watcharinyanon, A.A. Zakharov, L.I. Johansson, Phys. Rev. B 82 (2010) 205402.

[16] C. Virojanadara, A.A. Zakharov, S. Watcharinyanon, R. Yakimova, L.I. Johansson, New J. Phys. 12 (2010) 125015.

[17] V.C.Y. Kong, F.R. Foulkes, D.W. Kirk, J.T. Hinatsu, Int. J. Hydrogen Energy 24 (1999) 665.

[18] W. Grochala, P.P. Edwards, Chem. Rev. 104 (2004) 1283.

[19] W. Kern, D. Puotinen, RCA Rev. 31 (1970) 187.

[20] R. Nyholm, J.N. Andersen, U. Johansson, B.N. Jensen, I. Lindau, Nucl. Instr. \& Meth. in Phys. Res. A 467-468 (2001) 520.

[21] B.N. Jensen, S.M. Butorin, T. Kaurila, R. Nyholm, L.I. Johansson, Nucl. Instr. \& Meth. in Phys. Res. A 394 (1997) 243.

[22] M. Farjam, H. Rafii-Tabar, Phys. Rev. B 79 (2009) 045417.

[23] K. sugawara, K. Kanetani, T. Sato, T. Takahashi, AIP Advances 1 (2011) 022103.

[24] A.K.M.A. Islam, Phys. stat. sol. (b) 180 (1993) 9.

[25] K.V. Emtsev, F. Speck, T. Seyller, L. Ley, J.D. Riley, Phys. Rev. B 77 (2008) 155303.

[26] A.L. Walter, A. Bostwick, K.-J. Jeon, F. Speck, M. Ostler, T. Seyller, L. Moreschini, Y.J. Chang, M. Polini, R. Asgari, A.H. MacDonald, K. Horn, E. Rotenberg, Phys. Rev. B 84 (2011) 085410.

[27] A. Bostwick, F. Speck, T. Seyller, K. Horn, M. Polini, R. Asgari, A.H. MacDonald, E. Rotenberg, Science 328 (2010) 999. 


\section{Figure captions}

Fig. 1. a) Mirror image recorded from the in situ as grown monolayer graphene at an electron energy of $-0.4 \mathrm{eV}, \mathrm{b}) \mathrm{LEEM}$ image recorded after hydrogenation at $3.3 \mathrm{eV}$, and c) Mirror image recorded after Li deposition at $-1.6 \mathrm{eV}$. Figs. 1d)-f) show the corresponding LEED patterns.

Fig. 2. Electron reflectivity curves collected from a) the as grown monolayer graphene sample, b) after hydrogenation , c) after Li deposition and d) after annealing at $600{ }^{\circ} \mathrm{C}$.

Fig. 3. C 1s core level spectra collected at photon energy of $450 \mathrm{eV}$ from a) the as grown monolayer graphene, after hydrogenation, and after Li deposition. b) After subsequent annealing from 300 to $900{ }^{\circ} \mathrm{C}$.

Fig. 4. Si 2p core level spectra collected at photon energy of $190 \mathrm{eV}$ from a) the as grown monolayer graphene, after hydrogenation, and after Li deposition. b) After subsequent annealing from 300 to $900{ }^{\circ} \mathrm{C}$.

Fig. 5. Li 1s core level spectra collected, at a photon energy of $140 \mathrm{eV}$, from the hydrogenated graphene sample after Li deposition and subsequent annealing from 300 to $900{ }^{\circ} \mathrm{C}$.

Fig. 6. $\pi$-band dispersion around the $\bar{K}$ point recorded from a) the as grown monolayer graphene sample, and after b) hydrogenation, c) Li deposition, d) annealing at 300, d) at 400 and e) at $600{ }^{\circ} \mathrm{C}$. 


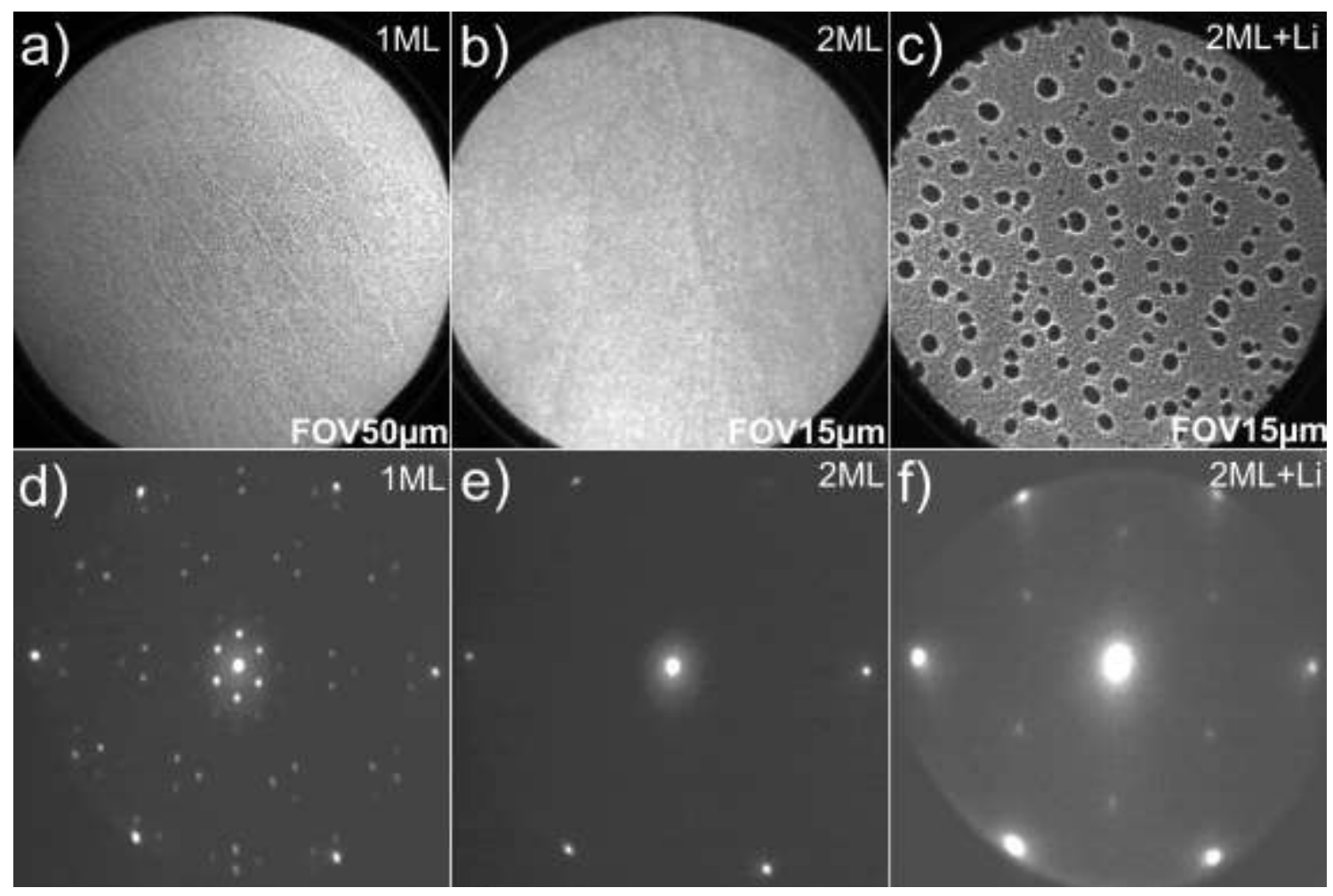

Fig. 1 


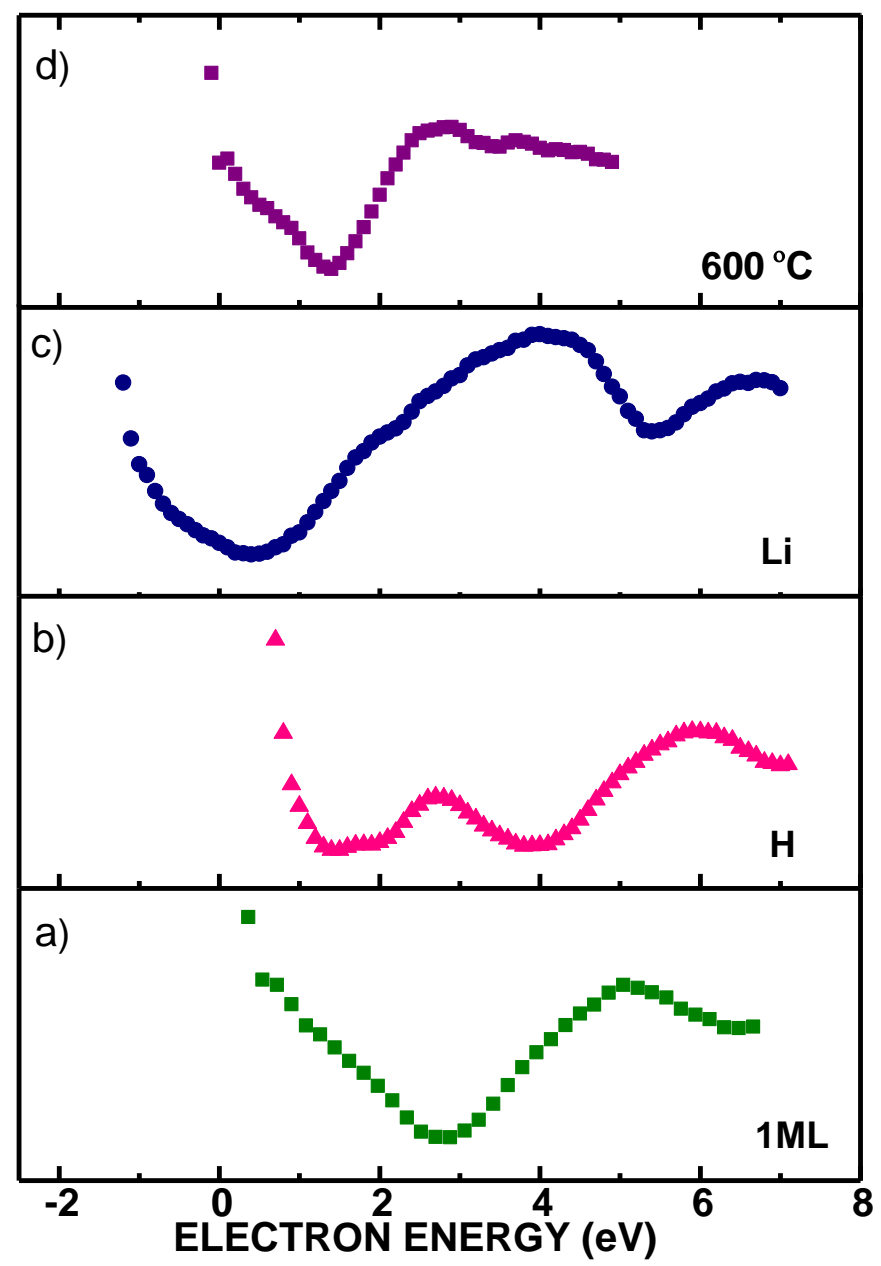

Fig. 2 


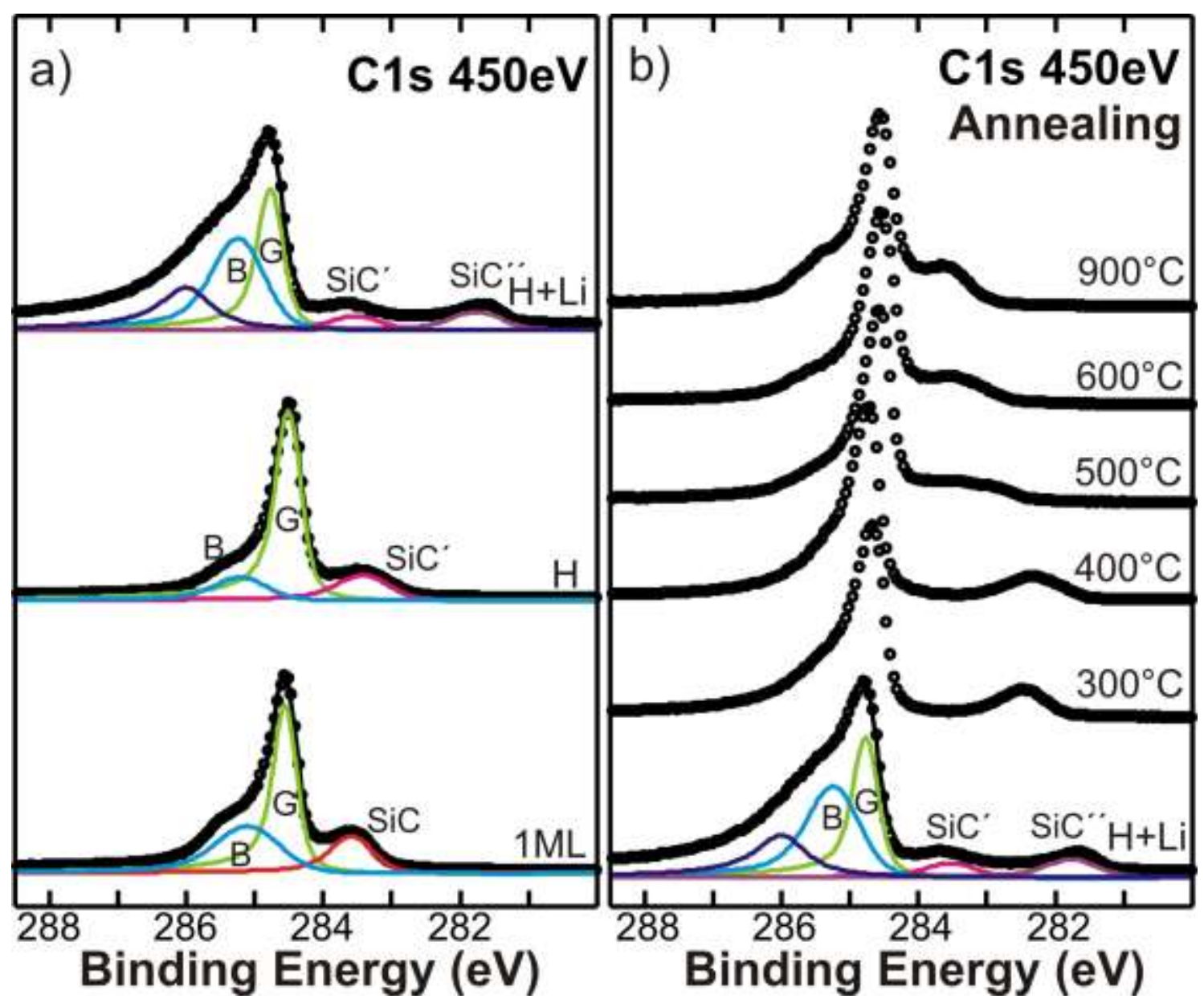

Fig. 3 


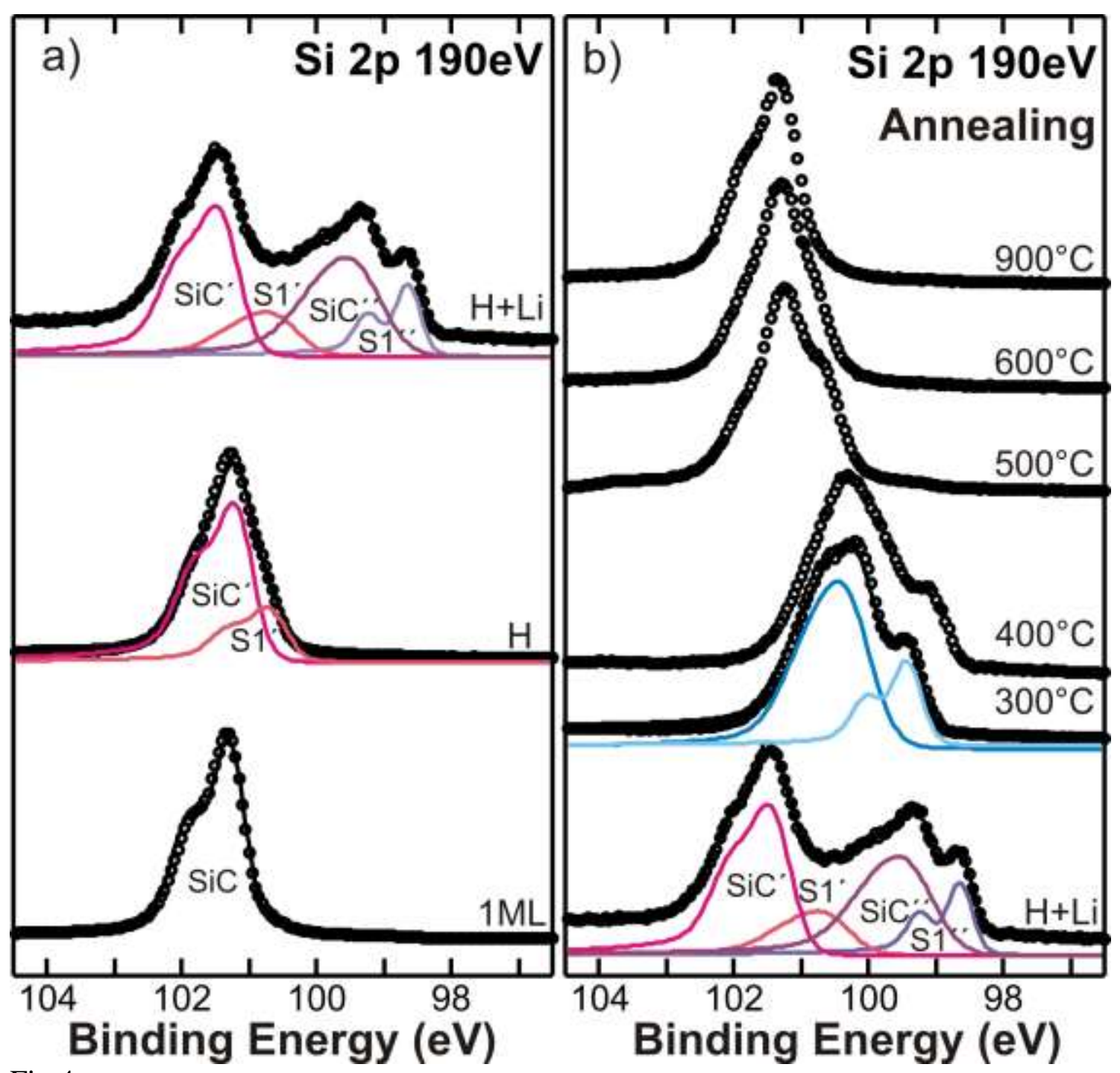

Fig.4 


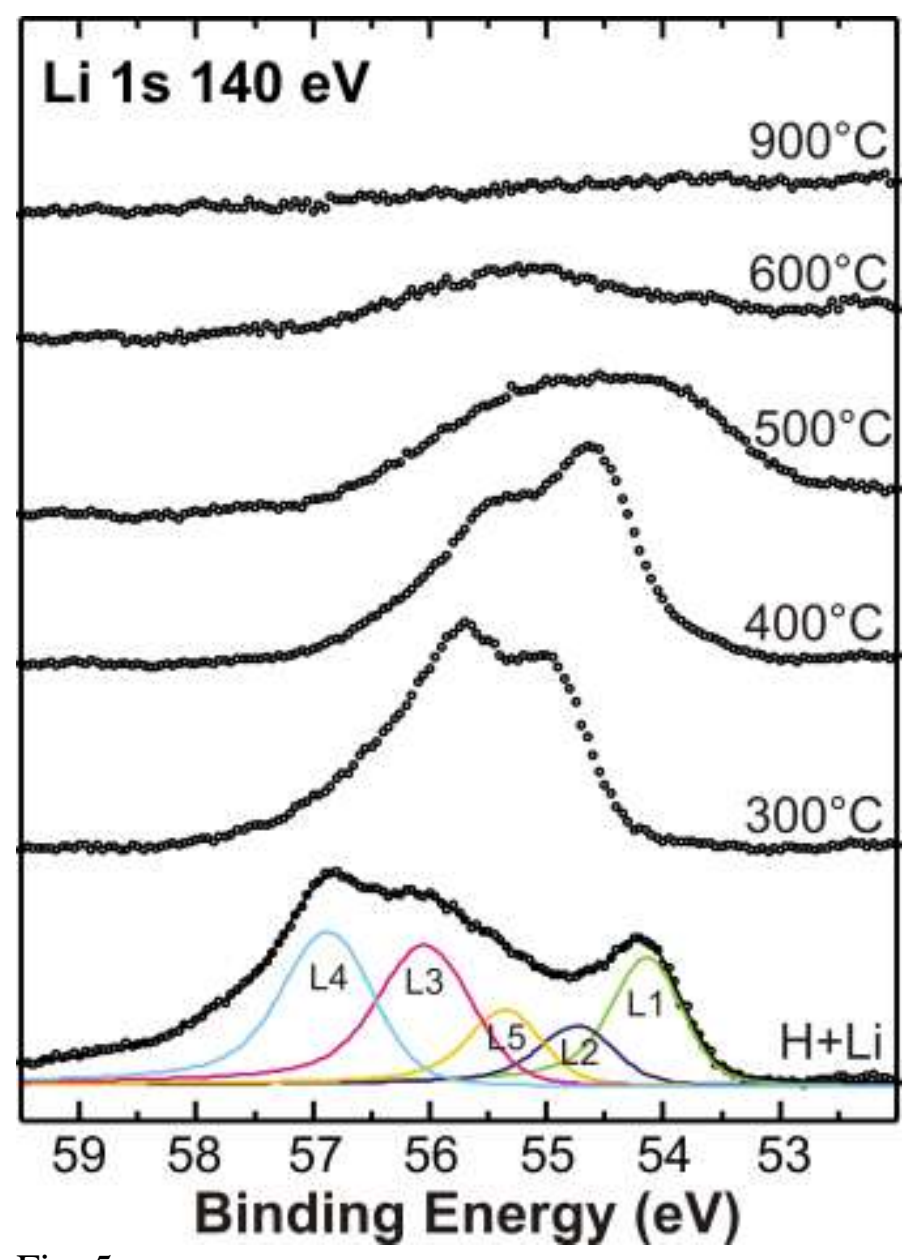

Fig. 5

a) $1 \mathrm{ML}$

b) $\mathrm{H}$

c) $\mathrm{H}+\mathrm{Li}$

d) $300^{\circ} \mathrm{C}$

e) $400^{\circ} \mathrm{C}$

f) $600^{\circ} \mathrm{C}$

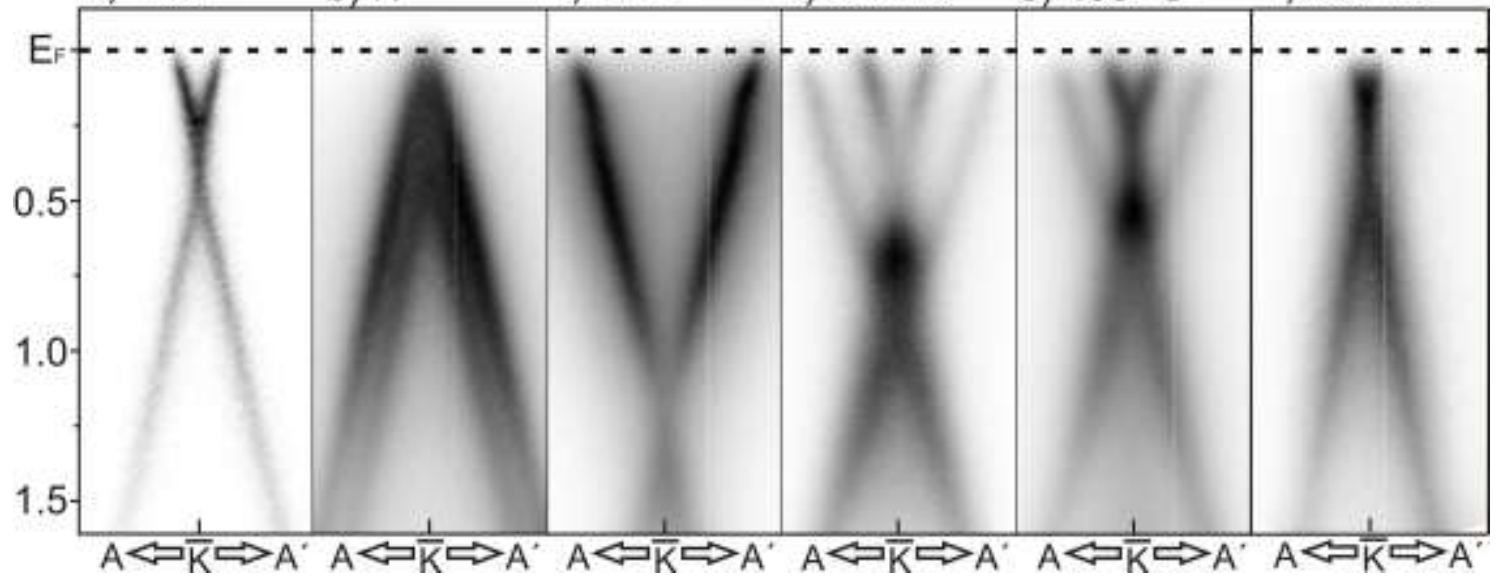

Fig. 6 